\title{
Progressive ocean wave modelling in drum centrifuge
}

\author{
F.P. $\mathrm{Gao}^{1,2}$ \& M.F. Randolph ${ }^{2}$ \\ ${ }^{I}$ Institute of Mechanics, Chinese Academy of Sciences, Beijing, P.R. China \\ ${ }^{2}$ Special Research Centre for Offshore Foundation Systems, The University of Western Australia, \\ Nedlands, Australia
}

\begin{abstract}
Ocean wave modelling in the centrifuge is a novel technique for the study of seabed response to waves and wave-seabed-structure interaction problems in offshore engineering. Based on the similarity analysis of wave simulation in centrifuge, a paddle-type wave generation system was established in a drum centrifuge. A variant of a double-beach wave absorber, made of porous metal plate covered with plastic mesh, was adopted for the purposes of wave absorption. Instrumentation comprised a wave height monitor in the centre of the water channel and pore pressure transducers in the base of the channel at two positions relative to the paddle device. Results from a series of tests are presented and show that the wave generation system was able to simulate progressive waves in shallow to intermediate water depths.
\end{abstract}

\section{INTRODUCTION}

Under severe ocean wave loading, sandy seabeds may undergo shear failure or liquefaction, which may cause instability of submarine pipelines, offshore platforms or other marine facilities. In the experimental investigation of the above problems, it is necessary to simulate ocean waves in the laboratory. The most common approach for wave simulation are flumes or tanks operating under $1 \mathrm{~g}$ conditions, but very large flumes or tanks would be required for most problems of practical significance unless the problems are scaled down. This can lead to difficulties in correctly simulating the seabed response, which is stress-level dependent.

Centrifugal experiments utilizing scaled models in a high gravity environment are accepted as the best approach to create the stress conditions appropriate for prototype conditions (Schofield 1980, Taylor 1995) and are particularly suitable for modelling gravity and time dependent problems. Wave simulation in the centrifuge is a key technique for carrying out the research mentioned above.

Tsunami-like solitary waves were first generated in the drum centrifuge at Cambridge University, by means of immersing a rectangular float into water (Sekiguchi \& Phillips 1991). Thereafter, standing waves and progressive waves were generated on the conventional beam centrifuge at Kyoto University (Sekiguchi et al. 1994, Sekiguchi et al. 1998). Considering the limited length of the test box in beam centrifuge, a slotted vertical partition was employed as a wave absorber for the purpose of progressive wave generation. Nevertheless, the efficiency of the slotted partition wave absorber is relative to the wavelength of the waves generated; only waves whose wavelength is about 4 times the distance between the partition and the far end wall are dissipated with low reflection coefficients, thus the wave frequency could not be changed during experiments in the beam centrifuge (Sekiguchi et al. 1998). Compared with the beam centrifuge, the drum centrifuge offers more advantages in many aspects, and in particular the longer testing area along its circumference, which is very helpful for water wave generation (Stewart et al. 1998).

In this paper, the principle of wave generation in centrifuge is presented first, based on which a new progressive wave generation system is described, as implemented in the drum centrifuge at the University of Western Australia. A series of progressive wave generation tests were conducted with the new facility and the measured results are compared with the theoretical predictions.

\section{MECHANISM OF WAVE GENERATION IN GEO-CENTRIFUGE}

\subsection{Similarity analysis for ocean wave modelling in centrifuge}

In the case of ocean waves with a free surface, gravitational effects predominate while the effects of other factors, such as viscosity, surface tension, etc, are generally small and can be neglected. It is well known 
Table 1. Similarity laws for wave modelling in a centrifuge based on Froude scaling.

\begin{tabular}{lll}
\hline Variable & Unit & $\begin{array}{l}\text { Scale } \\
\text { Factor }(\lambda)\end{array}$ \\
\hline Centrifugal acceleration $(a)$ & $\mathrm{LT}^{-2}$ & $N$ \\
Froude number $(F r)$ & 1 & 1 \\
Water depth $(d)$ & $\mathrm{L}$ & $1 / N$ \\
Wave length $(L)$ & $\mathrm{L}$ & $1 / N$ \\
Wave period $(T)$ & $\mathrm{T}$ & $1 / N$ \\
Wave velocity $(c)$ & $\mathrm{LT}^{-1}$ & 1 \\
Water particle velocity $(U)$ & $\mathrm{LT}^{-1}$ & 1 \\
Fluid (water) density $(\rho)$ & $\mathrm{ML}^{-3}$ & 1 \\
Wave pressure on seabed $\left(P_{o}\right)$ & $\mathrm{ML}^{-1} \mathrm{~T}^{-2}$ & 1 \\
\hline
\end{tabular}

that with water both Reynolds and Froude similitude cannot be achieved concurrently. For gravitational wave simulation, it is reasonable to employ the Froude scaling process with allowance made for variation in Reynolds number (Chakrabrarti 1994).

The Froude number $(F r)$, which physically indicates the ratio of inertial force to gravitational force, is defined as

$F r=\frac{U_{m}}{\sqrt{a D}}$

where $U_{m}$ is the maximum value of wave-induced water particle velocity, $a$ is the centrifugal acceleration, $D$ is a characteristic dimension (e.g. the water depth). According to Froude's law, the following scaling law should be maintained:

$\frac{\lambda_{U_{m}}}{\sqrt{\lambda_{a} \lambda_{D}}}=1$

where $\lambda$ represents the ratio of the model parameters to those of the prototype. In centrifuge tests, $\lambda_{D}=1 / N$ and $a=N g$, in which $N$ is the centrifugal g-level and $g$ is the gravitational acceleration. Therefore

$\lambda_{U_{m}}=1$

The wave-period scale can be deduced as

$\lambda_{T}=\lambda_{D} / \lambda_{U_{m}}=1 / N$

where $T$ is the wave period. The similarity laws for ocean wave modelling in a centrifuge can be deduced as listed in Table 1, in which $c$ is the wave velocity $(c=L / T)$.

\subsection{Theoretical background to the paddle-type wave maker}

For gravitational ocean waves, the fluid can be assumed to be homogeneous, inviscid, incompressible and its motion irrotational. As such, the motion of the fluid can be described by a velocity potential flow and the velocity potential satisfies the Laplace equation. Regular harmonic waves are commonly used for understanding the physics of fluid-structure interaction. According to wave generation theory (Hughes 1993), in the water region defined by $0 \geqslant y \geqslant-d$ as shown in Figure 1, the wave elevation $h(x, t)$ of the sinusoidal wave generated with paddle-type wave maker is

$$
\begin{aligned}
h(x, t)= & \frac{2 S \sinh (k d)}{\sinh (2 k d)+2 k d} \\
& \times\left[\sinh (k d)+\frac{1-\cosh (k d)}{k\left(d-d_{s}\right)}\right] e^{i(k x-\omega t)}
\end{aligned}
$$

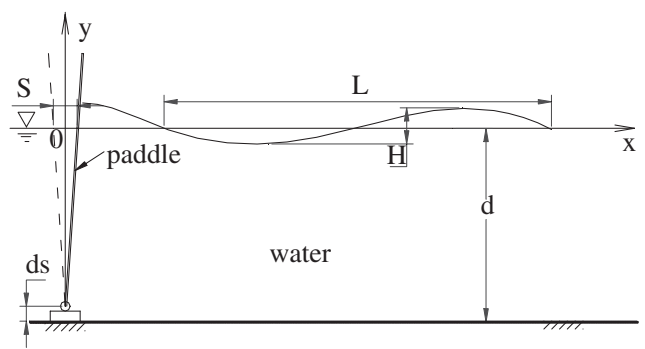

Figure 1. Sketch of paddle type wave maker.

where $S$ is the stroke of paddle at water surface, $\omega$ is the angular velocity of the wave generated by the paddle $(\omega=2 \pi / T), d$ is the water depth, $d_{s}(=0.02 \mathrm{~m})$ is the vertical distance from the seabed surface to the paddle hinge, $g$ is the acceleration of gravity, $k$ $(=2 \pi / L)$ is the wave number, and $L$ is the wave length. The wave dispersion equation is

$$
\omega^{2}=N g k \tanh (k d)
$$

From Equation (5), the wave height $H$ can be expressed as

$$
H=\frac{4 S \sinh (k d)}{\sinh (2 k d)+2 k d}\left[\sinh (k d)+\frac{1-\cosh (k d)}{k\left(d-d_{s}\right)}\right]
$$

\section{EXPERIMENTAL SETUP}

\subsection{Drum centrifuge}

The wave modelling experiments were conducted in a drum centrifuge at the University of Western Australia. The drum centrifuge features a channel for sample containment, $300 \mathrm{~mm}$ in width (measured vertically) and $200 \mathrm{~mm}$ in depth (measured radially), as shown in Figure 2. The channel has an outer diameter 




Figure 2. View of drum centrifuge with protective enclosure removed and control tool table in position.

$D=1.2 \mathrm{~m}$, and its maximum rotational speed is $850 \mathrm{rpm}$, equivalent to an acceleration of $480 \mathrm{~g}$ at the bottom of the channel. A novel and versatile tool table, which is located at the centre of the sample containment channel, can be stopped and started independently of the channel. A fast sampling data acquisition system, whose maximum sampling rate is $100 \mathrm{kHz}$, was designed for dynamic problems. The sample containment channel is also fitted with a fluid distribution system, to enable the channel to be filled with water or emptied easily (Stewart et al. 1998).

\subsection{Paddle-type wave maker in drum centrifuge}

There are several methods to generate progressive waves on the ground. However, the design of the device for wave generation in the centrifuge must take account of the high centrifugal acceleration environment and the corresponding high frequency of the waves to be generated. For these reasons, a paddle-type wave maker was adopted, the key parts comprising a cam wheel driven by a servo DC motor, a paddle hinged at the bottom of centrifuge channel, and a crank shaft to link the paddle and cam wheel, as shown in Figure 3.

The lengths of the components of the wave maker were designed based on the crank-guide mechanism, to achieve a maximum paddle-swaying angle of about $18^{\circ}$. The radius of the cam wheel is changeable from $10 \mathrm{~mm}$ to $24 \mathrm{~mm}$ with an interval of $2 \mathrm{~mm}$, by means of screw holes with various offset distance on the cam wheel. A closed-loop servo-controlled system, using a DC tacho mounted directly on the motor shaft, provides close control of the motor rotation speed. The servo-motor must provide sufficient torque to overcome the following main loads:

1. wave loading on paddle;

2. inertia force on paddle and crank shaft;

3. centrifugal force on crank shaft.

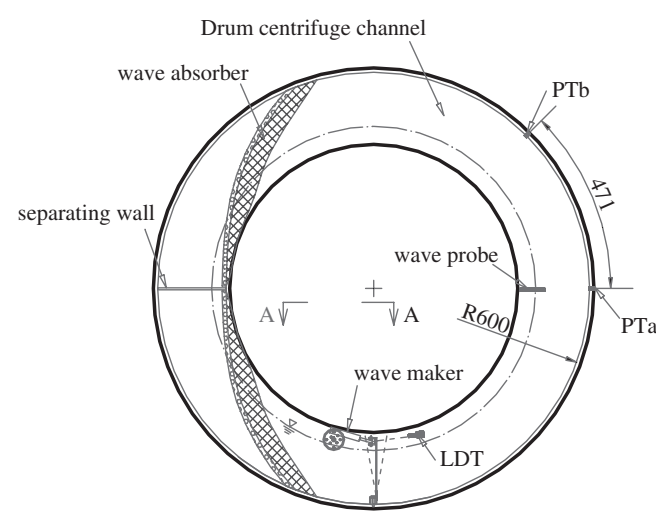

(a) Plan view of the wave generation system

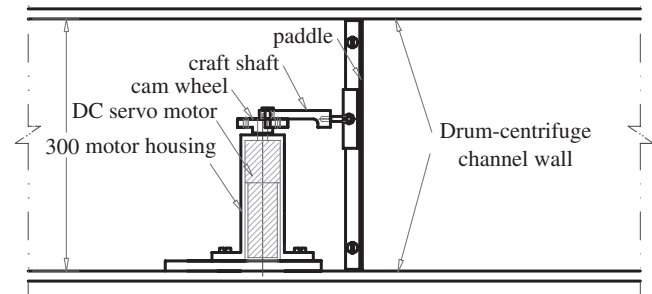

(b) A-A section view for the paddle-type wave maker (enlarged)

Figure 3. Arrangement of wave generation system in drum-centrifuge (dimensions in $\mathrm{mm}$ ).

A Maxon DC Servo Motor was chosen, with maximum continuous torque of $2.94 \mathrm{Nm}$, and maximum intermittent torque of $5.88 \mathrm{Nm}$. Thus the paddle can sway with a given frequency to obtain the desired wave height. The movement of the paddle is monitored with a Laser Displacement Transducer (LDT), as shown in Figure 3(a).

\subsection{Wave absorber design}

The incident waves generated by the wave maker must be dissipated in the drum centrifuge channel without reflection in order to achieve high quality data. Many different types of wave absorbers have been tried in wave flumes and basins, with varying degrees of success. They can be divided into two types: (1) caissontype and (2) beach-type.

The caisson-type absorber consists of a slotted vertical front partition and an internal chamber behind the partition. In this type of absorber, transformation of the wave energy into turbulence, produced by the narrowness of the front apertures, plays an important part in the wave dissipation phenomenon. In order to achieve high wave absorption efficiency, rectangular 
vertical apertures are distributed over a quarter to a third of the front partition.

Since one of the main advantages of this type of absorber over a beach-type absorber is the very short length, it was chosen for use in beam centrifuge tests, where the box length was only $0.55 \mathrm{~m}$ (Sechiguchi et al. 1998). It was found, however, that this kind of absorber achieved an efficiency of $90 \%$ or more only when the distance between the partition and the back wall of the chamber was about a quarter of the wavelength. This is a disadvantage if the absorber is required to cope with a wide range of wave frequencies.

The beach-type absorber is simpler and more common, compared with the caisson-type absorber. The main working principle of this type of absorber is dissipation of the wave energy by the waves breaking on a gently sloping plane of significant length. Wave absorbing beaches have the advantage of simple design and of being very efficient for a wide range of wave frequencies. However, their large size prohibits use in a conventional beam centrifuge.

Many solutions have been proposed to minimise the size of the wave absorbing beaches under 1-g conditions. Constructions combining the slope of absorbing beaches and porous walls increase the efficiency and also reduce the size (Ouellet \& Datta 1987). As a general rule it has been found that combining several wave absorbing energy effects leads to an important reduction in the required size.

Even though the channel of the drum centrifuge is much longer than the sample box for a beam centrifuge, its limited space still necessitates the use of an absorber with minimum length and slopes that are steeper than optimum. The benefits of using permeable material in the sloping beach are well known in both field and laboratory conditions. In order to maximise the efficiency over a wide range of wave conditions, a special wave absorber was designed, based on a double beach-type wave absorber made of porous metal plate covered with plastic mesh (Figs. 3, 4).

Due to the swaying motion of the paddle, two sets of waves are generated travelling in opposite directions around the channel. A separating wall is mounted at the middle of the double beach, to avoid the two series waves affecting each other. Only the waves generated in the longer part of the channel are used for the experiments. The purpose of using porous metal sheet and plastic mesh was to increase the wave breaking phenomenon and the viscous dissipation process of the wave energy. The slope of each porous beach was variable to reduce the total required length of the beach. Besides the common advantages over caisson-type absorbers, another main advantage of the new absorber is its shorter length than conventional beaches. The efficiency of the absorber will be discussed in Section 4.2.

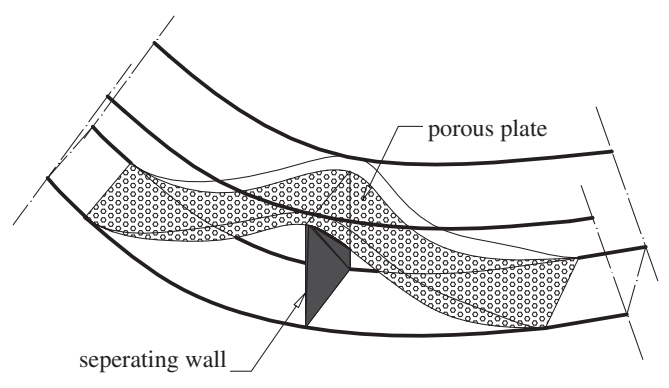

Figure 4. Sketch of porous beach type absorber.

\subsection{Testing procedure}

The following testing procedure was adopted to generate progressive water waves in the drum centrifuge:

1. firstly, the wave maker, wave absorber and all transducers were installed in drum centrifuge channel;

2. the centrifuge was then accelerated to a pre-set g-level, such as $50 \mathrm{~g}$;

3 . the centrifuge channel was filled with water to a given height (e.g. $140 \mathrm{~mm}$ ) through the fluid distribution system;

4. thereafter, the DC servo-motor was started at a predetermined frequency (e.g. $10 \mathrm{~Hz}$ ) to generate the waves;

5. the fast data acquisition system was then employed to sample the wave height and the wave-induced pressures on the seabed, the latter measured with two pressure transducers, PTa and PTb, as shown in Figure 3.

For the measurement of the wave height, a capacitance-type wave probe is employed, which is located above PTa as depicted in Figure 3. In addition, the movement of the paddle was checked by a laser displacement transducer (LDT), which was installed on the centrifuge channel.

\section{EXPERIMENTAL RESULTS AND DISCUSSIONS}

\subsection{Typical experimental results}

Typical experimental results are shown in Figure 5. The wave was generated under conditions: $a=50 \mathrm{~g}$, $d=0.12 \mathrm{~m}$. Note that the g level quoted is that at the bottom of the channel. The wave frequency is $8.6 \mathrm{~Hz}$. It can be seen from Figure 5 that the pressure amplitudes at PTa and PTb are practically the same. The ratio of wave propagation period between $\mathrm{PTa}$ and $\mathrm{PTb}(\delta t)$ to wave period $(T)$ is $\delta t / T=0.08 / 0.12=0.67$. Based on the dispersion equation (6), the wavelength can be obtained as $L=0.68 \mathrm{~m}$. Thus the ratio of the distance between PTa and PTb $(\delta l)$ to the wavelength $(L)$ is 


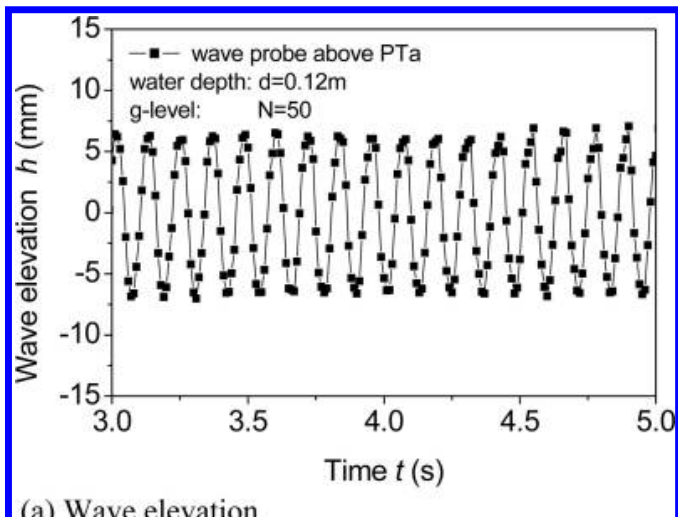

(a) Wave elevation

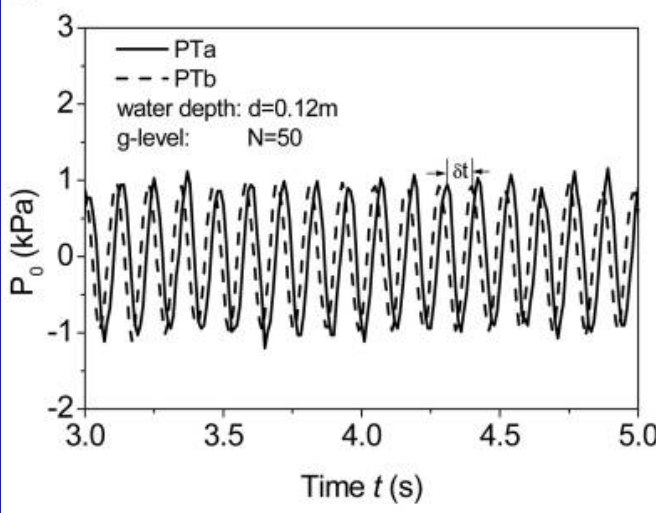

(b) Wave induced pressures at PTa and PTb

Figure 5. Typical wave generation experiment results.

$\delta l / L=0.47 / 0.68=0.69$. The agreement between the time and distance proportions therefore confirms that the water waves generated are consistent with a progressive pattern.

\subsection{Comparison with theoretical results}

Several theories exist for computing wave-induced pressures on the seabed. These include the Airy (linear) wave theory, the second order Stokes' wave theory and higher order wave theories. Stokes' theory gives a multi-order approximation to the exact solution of the differential equations describing wave behaviour and is a finite amplitude wave theory. The choice of an appropriate theory for a particular situation is determined by the wave characteristics: wave height $H$, wave period $T$, water depth $d$ and gravitational acceleration. In the centrifuge tests, gravitational acceleration is replaced by the centrifugal acceleration $a$. The relevant characteristics are grouped into two governing dimensionless parameters: $d / a T^{2}$ and $H / a T^{2}$ (Dean 1994).

The wave modelling experiments were conducted at various water depths (up to $12 \mathrm{~m}$ prototype), wave
Table 2. Results of wave modelling in drum centrifuge.

\begin{tabular}{llllrll}
\hline $\begin{array}{l}\text { Test } \\
\text { No. }\end{array}$ & $N_{b}$ & $\begin{array}{l}d \\
(\mathrm{~m})\end{array}$ & $\begin{array}{l}f \\
(\mathrm{~Hz})\end{array}$ & \begin{tabular}{l}
\multicolumn{1}{l}{$(\mathrm{mm})$} \\
$(\mathrm{m})$
\end{tabular} & $\begin{array}{l}L \\
(\mathrm{kPa})\end{array}$ \\
\hline 1 & 50 & 0.12 & 5.08 & 7.1 & 0.66 & 1.01 \\
2 & 50 & 0.12 & 6.84 & 9.2 & 0.65 & 1.03 \\
3 & 50 & 0.12 & 8.60 & 12.6 & 0.55 & 1.05 \\
4 & 50 & 0.15 & 6.45 & 18.3 & 0.67 & 1.31 \\
5 & 50 & 0.15 & 7.62 & 20.2 & 0.62 & 1.26 \\
6 & 80 & 0.12 & 6.84 & 8.3 & 0.71 & 1.54 \\
7 & 80 & 0.12 & 8.40 & 9.1 & 0.63 & 1.50 \\
8 & 80 & 0.15 & 7.62 & 15.4 & 0.60 & 2.10 \\
9 & 80 & 0.15 & 8.02 & 15.6 & 0.52 & 2.31 \\
\hline
\end{tabular}

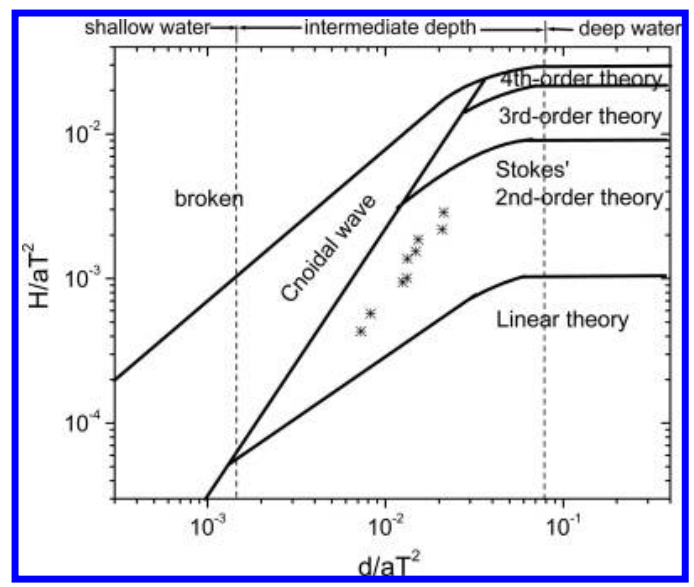

Figure 6. Regions of validity for available wave theories.

frequency and g levels. The measured wave parameters in the experiments are listed in Table 2, with measured wave heights of up to $1.2 \mathrm{~m}$. Guided by the two governing dimensionless groups above, the appropriate theory can be chosen from those available. As indicated in Figure 6, Stokes' 2nd-order theory is the appropriate one for the present tests, so that the expression for the amplitude of wave-induced pressure on an impermeable rigid horizontal bottom is

$$
\begin{aligned}
P_{0}= & \frac{\rho N g H}{2 \cosh (k d)}+\frac{3}{16} \rho N g k H^{2} \frac{\tanh (k d)}{\sinh ^{2}(k d)} \times \\
& {\left[\frac{1}{\sinh ^{2}(k d)}-\frac{1}{3}\right]-\frac{1}{16} \rho N g k H^{2} \frac{\tanh (k d)}{\sinh ^{2}(k d)} }
\end{aligned}
$$

in which $N$ is chosen as the effective g level, which is related to the g-level at the base of drum centrifuge channel $N_{b}$ by $N=(D-2 d / 3) N_{b}$. The theoretical wave-induced pressures corresponding to the measured wave height, wavelength and wave frequency are calculated from Equation (8). With the measured pressure as ordinate value and the theoretical prediction 


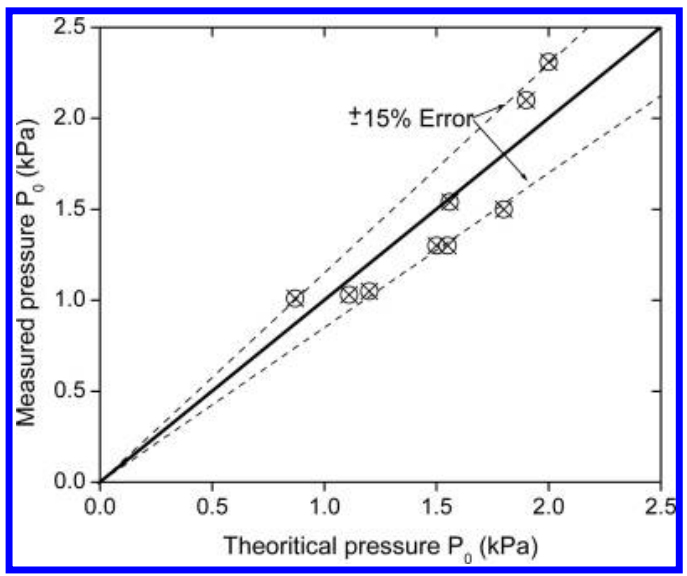

Figure 7. Comparison of measured wave pressures with theoretical predictions.

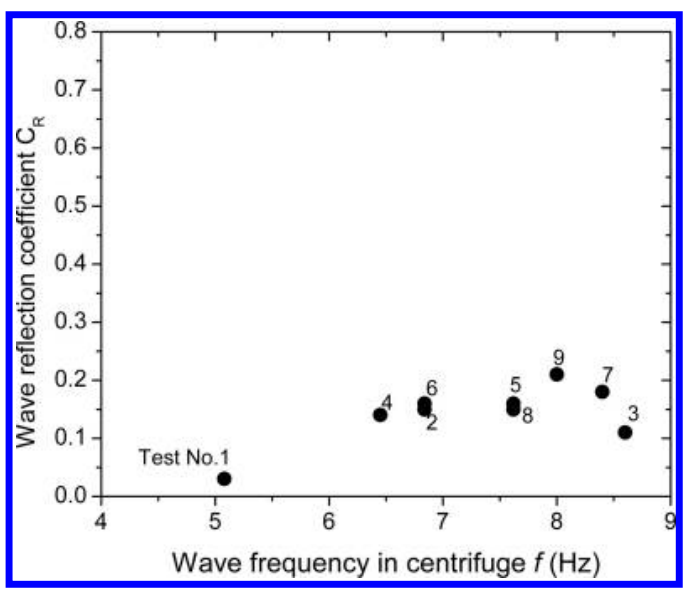

Figure 8. Wave reflection coefficients.

as abscissa, the data points are then plotted in Figure 7. As indicated in this figure, most of the test data points lie within about $\pm 15 \%$ error range from the $45^{\circ}$ solid line. The measured values and the theoretical predictions are in good agreement.

In addition, the wave reflection coefficients can be obtained using the method of Goda et al. (1976), as shown in Figure 8. Unlike the vertical partition wave absorber used in the beam centrifuge, the wave reflection coefficients of the beach-type absorber are normally less than $20 \%$, indicating that the wave absorber is efficient over a wide range of wave frequencies.

\section{CONCLUSIONS}

Based on a similarity analysis for modelling progressive waves, a new wave generation system was established on a drum centrifuge. Comparison of the phase shift in measured pore pressure changes and the distance between the transducers confirmed the progressive nature of the waves. A novel double beachtype wave absorber was found to provide an efficient solution for the absorption of waves over a range of frequencies. The experimental results show that the wave generation system is capable of modelling progressive ocean waves in intermediate to shallow water zones, with wave heights of up to $10 \%$ of the water depth. The device can be employed for further applications in experimental research on wave-structureseabed interaction.

\section{ACKNOWLEDGEMENTS}

Funding from the Australian Research Council (Grant No. A00104092) is gratefully acknowledged. We are also grateful to Bart Thompson who provided general assistance with assembly, testing and operation, and Clem Ryan carried out the electronics work on the servo-motor control and other parts of the drum centrifuge. The first author is also grateful for the support from 'Tenth Five-year Plan' of Chinese Academy of Sciences (Grant No. KJCX2-SW-L03).

\section{REFERENCES}

Chakrabarti, S.K., 1994. Offshore Structure Modeling. JBW Printers \& Binder Pte. Ltd.

Dean, R.G., Dalrymple, R.A., 1994. Water Wave Mechanics for Engineers and Scientists. World Scientific, Singapore.

Goda, Y. and Suzuki, Y., 1976. Estimation of incident and reflected wave in random wave experiments with reflecting coastal structures. Proceedings, Fifteenth Coastal Engineering Conference, ASCE, Vol.1, 828-845.

Hughes, S.A., 1993. Laboratory wave generation. In: Physical Models and Laboratory Techniques in Coastal Engineering, World Scientific Publishing Co., 333-457.

Ouellet, Y., Datta, I., 1987. A survey of wave absorbers. Journal of Hydraulic Research, 24 (4): 265-280.

Schofield, A.N, 1980. Cambridge geotechnical centrifuge operations. Géotechnique, 30(3), 227-268.

Sekiguchi, H., Kita, K., Okamoto, O., 1994. Wave-induced instability of sand beds. Proceedings, International Conference CENTRIFUGE 94, Singapore, 1994: 295-300.

Sekiguchi, H., Kita, K., Sassa, S., and Shimamura, T., 1998. Generation of Progressive Fluid Waves in a Geo-Centrifuge. Geotechnical Testing Journal, ASTM, 21(2): 95-101.

Sekiguchi, H., Phillips, R., 1991. Generation of water waves in a drum centrifuge. Proceedings, International Conference CENTRIFUGE 91, Colorado, 343-350.

Stewart, D.P., Boyle, R.S. and Randolph, M.F., 1998. Experience with a new drum centrifuge. In: Proceedings, International Conference CENTRIFUGE 98, Tokyo, 35-40.

Taylor, R.N, 1995. Centrifuges in modelling: Principles and scale effects. Geotechnical Centrifuge Technology, Blackie Academic \& Professional, 19-33. 